\title{
Datación de restos arqueológicos encontrados en Checua (Cundinamarca - Colombia) mediante resonancia paramagnética electrónica
}

\author{
Eduar Carvajal' ${ }^{1}$, Luis Montes ${ }^{2}$, Ovidio A. Almanza ${ }^{1, *}$ \\ ${ }^{1}$ Departamento de Física, Grupo de Física Aplicada, Universidad Nacional de Colombia, Bogotá, Colombia \\ ${ }^{2}$ Departamento de Geociencias, Universidad Nacional de Colombia, Bogotá, Colombia
}

\begin{abstract}
Resumen
En este trabajo se presentan los resultados obtenidos al datar por medio de resonancia paramagnética electrónica (RPE) muestras de esmalte dental humano provenientes del sitio arqueológico de Checua, situado a $5^{\circ} 04^{\prime}$ de latitud norte y a $73^{\circ} 53^{\prime}$ de longitud este, a una altitud de $3.600 \mathrm{~m}$ sobre el nivel del mar, y a $60 \mathrm{~km}$ al noreste de Bogotá. El esmalte dental se irradió con rayos gama y los radicales libres producidos se cuantificaron usando un espectrómetro de RPE, banda X. A partir de la curva de intensidad de la señal RPE, comparada con la dosis absorbida por el esmalte, se hizo un ajuste que permitió obtener la dosis media arqueológica acumulada en el esmalte $\left(\mathrm{D}_{\mathrm{E}}\right)$ durante el periodo de enterramiento de la muestra. El valor obtenido fue de 3,0 $\pm 0,08 \mathrm{~Gy}$. Para estimar la edad de la muestra se usó el programa computacional ROSY, obteniendo una edad media del individuo de $5.021 \pm 202$ años antes del presente. La edad de las muestras corresponde a seres humanos que vivieron en el periodo cuaternario, en la época del holoceno. Los resultados obtenidos muestran el potencial de la técnica RPE como herramienta de datación de muestras cuaternarias en Colombia y abre la posibilidad de usarla en otro tipo de muestras como estalagmitas, calcitas, conchas de moluscos y corales. Este trabajo es el tercero realizado en Colombia en este campo después del trabajo de tesis de Sandoval y Carvajal (Sandoval 2010, Carvajal 2011 a,b).
\end{abstract}

Palabras clave: EPR, esmalte dental, datación arqueológica.

Electron paramagnetic resonance dating of archaeological rests from Checua (Cundinamarca - Colombia)

\begin{abstract}
This paper presents the results obtained from using electron paramagnetic resonance (EPR) to analyse human tooth enamel found at the Checua archaeological site (Cundinamarca, Colombia), located $5^{\circ} 04^{\prime}$ latitude north and $73^{\circ} 53^{\prime}$ longitude east, 3,600 m above sea level and $60 \mathrm{~km}$ northeast of Bogotá. The tooth enamel was irradiated with gamma rays and the resulting free radicals were measured using an electron spin resonance (ESR) X-band spectrometer. Comparing the RPE signal intensity curve to the absorbed dose, it was possible to adjust this curve, which allowed us to obtain the mean archaeological dose accumulated in the enamel $\left(\mathrm{D}_{\mathrm{E}}\right)$ during the period it was buried. The resulting value was $3.20 \pm 0.08 \mathrm{~Gy}$. By using the ROSY software, the mean age was estimated at $5021 \pm 202$ years before present (BP). This age corresponds to human beings who lived in the Quaternary period, Holocene epoch. These results show the EPR technique potential as a tool for dating Quaternary samples in Colombia and open the possibility of using it in stalagmite, calcite, mollusc shell and coral reef samples. This study is the third conducted in Colombia after the thesis work by Carvajal and Sandoval (Sandoval, 2010, Carvajal, 2011).
\end{abstract}

Key words: EPR/ESR, tooth enamel, Colombia, archaeological dating.

\section{Introducción}

El hombre siempre ha mostrado gran interés por su origen y su desarrollo a través de los tiempos, preguntándose sobre el qué, el cómo, el dónde y el cuándo de tal evolución. La respuesta al cuándo, ya sea de manera relativa o absoluta, le corresponde al campo de la datación. La datación permite que las interpretaciones arqueológicas se ubiquen en un espacio y un tiempo determinados de forma útil y coherente; para ello, los fenómenos físicos, químicos y geológicos que se dieron en el pasado se aprovechan para fechar eventos y materiales y escribir la historia de la tierra y sus habitantes (Ikeya, 1993).

Los diversos métodos de datación se basan en fenómenos como el decaimiento radiactivo, la concentración de defectos por radiación natural y las reacciones químicas. Todos 
ellos buscan determinar la edad de la muestra. El más conocido y convencional de estos métodos es el de datación por radiocarbono (carbono-14, ${ }^{14} \mathrm{C}$ ); recientemente, sin embargo, se ha utilizado la técnica de RPE en dosimetría y arqueología con base en el hecho de que la radiación ionizante produce centros paramagnéticos o radicales libres que pueden detectarse mediante dicha técnica y que, además, estos centros gozan de una vida media bastante prolongada en diversos materiales (más de 100 millones de años). Así pues, la concentración de centros en una muestra dada es una medida de la dosis total de radiación a la que ha sido expuesta. Este efecto puede emplearse para determinar los tiempos de exposición y, por ende, el tiempo de enterramiento de una muestra en algunos de los casos (Jonas, 1997).

La técnica RPE es muy confiable en muestras con una antigüedad de hasta 2 millones de años y no tiene el riesgo de trabajar en el límite de saturación de las muestras por efectos de la radiación. Las muestras de estalactita, conchas, corales, hueso, cuarzos, feldespatos y esmalte dental, por ejemplo, se datan mediante la RPE debido a su capacidad de acumular radicales libres por efecto de las radiaciones (Ülkü, 2004, Watanabe, et al., 2003, Renfrew, et al., 1992, Jonas, 1997, Walker, 2005, Grun, 1989, 2006).

El presente trabajo se propuso datar mediante resonancia paramagnética electrónica (RPE) muestras arqueológicas de restos fósiles de humanos encontradas en el sitio arqueológico de Checua, Cundinamarca, Colombia, donde, según la información disponible, habitaron cazadores recolectores entre los 8.500 y 3.000 años antes del presente (Groot, 1992). Una datación más exacta de los restos humanos encontrados es de vital importancia para reconstruir nuestro pasado y tener mayor información sobre la forma de vida de quienes habitaron nuestro territorio. La figura 1 muestra el maxilar inferior de uno de los cuerpos hallados en el entierro datado en este trabajo.

\section{Aspectos teóricos}

El esmalte dental está compuesto principalmente por un componente inorgánico llamado hidroxiapatita $\left(\mathrm{Ca}_{10}\left(\mathrm{PO}_{4}\right)_{6}\right.$ $\left.(\mathrm{OH})_{2}\right)$, el cual constituye entre 95 y $98 \%$ del esmalte. Es el material más mineralizado, estable y duro que posee el cuerpo humano, lo que lo hace útil para la datación de restos arqueológicos de humanos y de animales. En la hidroxiapatita pueden existir sustituciones isomórficas o impurezas de carbonatos (principalmente $\mathrm{CO}_{3}^{2-}$ ), con la capacidad de reemplazar los fosfatos o hidróxidos que la constituyen. Cuando inciden sobre materiales arqueológicos y geológicos como el esmalte dental, los diferentes tipos de

\footnotetext{
*Correspondencia:

Ovidio A. Almanza, oaalmanzam@unal.edu.co

Recibido: 23 de abril de 2014

Aceptado: 12 de junio de 2014
}

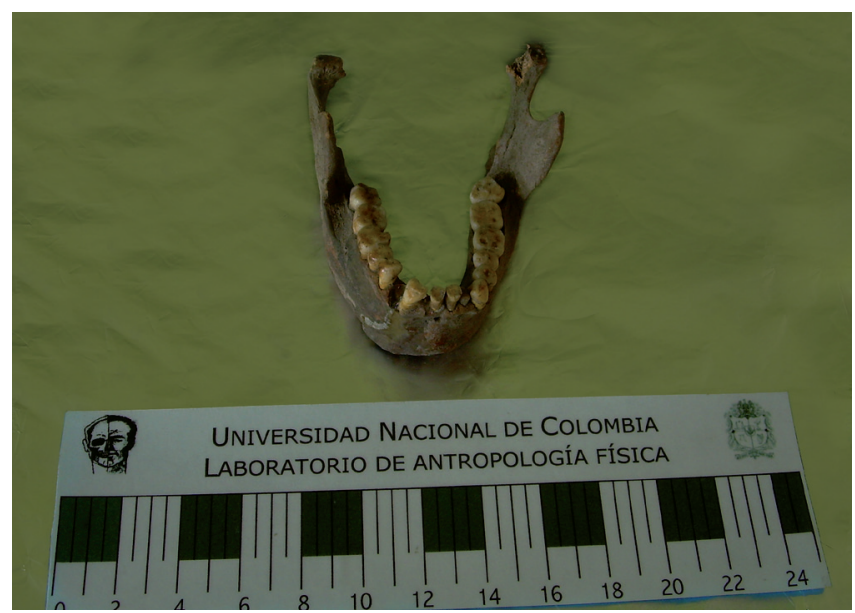

Figura 1. Parte de maxilar hallado en el entierro localizado en la región de Checua, Cundinamarca, Colombia, a partir del cual se extrajo un molar para el estudio por RPE

radiación ionizante presentes en el ambiente (rayos $\mathrm{X}$ o rayos gama provenientes de radionúclidos del uranio $\left({ }^{238} \mathrm{U}\right.$ y $\left.{ }^{235} \mathrm{U}\right)$, del torio $\left({ }^{232} \mathrm{Th}\right)$ y de sus radionúclidos hijos, y del potasio $\left.\left({ }^{40} \mathrm{~K}\right)\right)$ generan radicales del tipo $\mathrm{CO}_{2}^{-}, \mathrm{CO}_{3}^{-}, \mathrm{CO}^{-}, \mathrm{CO}_{3}^{3-}$ en estos carbonatos, los cuales pueden ser plenamente detectados mediante RPE. Sin embargo, algunos de ellos no son lo suficientemente estables en el tiempo para ser usados en la datación, excepto el radical $\mathrm{CO}_{2}^{-}$, cuyo tiempo de vida es de $10^{7}$ años (AIEA, 2002). Por lo tanto, el radical $\mathrm{CO}_{2}^{-}$juega el papel fundamental en la datación y la dosimetría del esmalte dental por RPE. Numerosos trabajos han reportado el uso de la señal de este radical para datar fósiles humanos, e incluso de animales como mamuts y venados (Ikeya, 1993).

La intensidad de la señal captada por RPE es proporcional a la concentración de espines, la cual, a su vez, es proporcional a la dosis de radiación a la que ha sido expuesta la muestra, a la tasa de dosis de radiación y al tiempo transcurrido desde su formación. El concepto de datación por RPE se basa en la determinación de la dosis total de radiación natural recibida por la muestra desde el momento de su muerte hasta el momento de su hallazgo (periodo de enterramiento), la cual se mide en Gy. La dosis total también recibe el nombre de dosis arqueológica o de dosis equivalente $\left(D_{\mathrm{E}}\right)$, dependiendo del campo de aplicación (geología o arqueometría).

La $D_{\mathrm{E}}$ depende de la tasa de dosis media anual de radiación natural $(\langle D>)$, que es la rapidez promedio con la que el material recibe la radiación natural en un año medida en Gy/año. Así, la edad ( $t$ ), o tiempo de enterramiento de una muestra, se determina por RPE con base en la razón entre $D_{E} \mathrm{y}<D>$ :

$$
t=\frac{D_{E}}{<D>}
$$


Las ventajas de la datación por RPE radican en que las mediciones pueden hacerse sin tener que calentar el material, lo que facilita el estudio de materiales orgánicos. Es decir, la técnica RPE no es destructiva y la medición de una misma muestra puede hacerse una y otra vez.

Para obtener la edad de una muestra por RPE se necesita, entonces, encontrar dos parámetros importantes: la dosis acumulada, $D_{E}$, y la tasa de dosis anual, $\langle D>$, durante el periodo de enterramiento. La $D_{E}$ puede obtenerse por el método de dosis aditiva, en el que la intensidad de la señal de RPE se calibra de acuerdo a la dosis de radiación. Para esto, alícuotas de la muestra se irradian paso a paso, con diferentes dosis y con fuentes de irradiación artificial como ${ }^{60} \mathrm{Co}$ o ${ }^{137} \mathrm{Cs}$, con el fin de detectar la dependencia de la intensidad de la RPE con respecto a la dosis absorbida. Al graficar esta dependencia, conocida como curva de respuesta de dosis, se extrapola a la dosis cero y, de esta forma, se obtiene la $D_{E}$.

Por otra parte, la $<D>$ no es necesariamente constante en el tiempo y se obtiene a partir del análisis de los elementos radioactivos en la muestra y sus alrededores, y de la contribución de la radiación cósmica (Ikeya, 1993; Jonas, 1997; Walker, 2005; Grun, 1989). La $<D>$ puede obtenerse experimentalmente si se conoce la concentración en parte por millón (ppm) de los principales elementos radiactivos, ${ }^{238} \mathrm{U},{ }^{232} \mathrm{Th}$ y ${ }^{40} \mathrm{~K}$, del sedimento alrededor de la muestra. Comúnmente, la concentración de estos elementos se determina mediante la espectrometría gama o el análisis de activación de neutrones (AAN); más recientemente, también se ha usado la espectrometría de masas (Ikeya, 1993). La contribución de la radiación cósmica puede determinarse mediante la ecuación para la datación por luminiscencia y por RPE (Prescott y Hutton, 1994):

$$
D=D_{0}\left[F+\mathrm{Je}^{\frac{\mathrm{h}}{\mathrm{H}}}\right]
$$

donde $D_{0}$ es la tasa de dosis cósmica al nivel del mar en una latitud geomagnética de 8,1 y a una altitud h $(2.600 \mathrm{~m})$. F, $\mathrm{J}$ y $\mathrm{H}$ son valores predeterminados que varían de acuerdo a la latitud geomagnética del sitio arqueológico en cuestión y pueden tomarse de Prescott y Hutton (1994).

La $D_{0}$ puede calcularse a partir de la profundidad de la muestra por medio de la siguiente ecuación (Prescott, et al., 1994):

$$
D_{0}=0.21 e^{\left(-0.070 x+0.0005 x^{2}\right)}
$$

En esta ecuación, la $D_{0}$ se expresa en mGy/año y $x$ representa la profundidad de la muestra expresada en $\mathrm{hgcm}^{-2}\left(100 \mathrm{gcm}^{-}\right.$ ${ }^{2}$ ). En nuestro caso, la profundidad a la que se encontró el entierro corresponde a $35 \mathrm{~cm}$, lo que equivale a $0,93 \mathrm{hgcm}^{-2}$ (Prescott y Hutton, 1994). Usando esta expresión, el valor encontrado para $D_{0}$ fue de $0,20 \pm 0,01 \mathrm{mGy} / \mathrm{a}$. La latitud geomagnética $(\lambda)$ del sitio se calculó usando la expresión:

$$
\operatorname{Sen} \lambda=0.203 \cos \theta \cos (\varphi-291)+0.979 \operatorname{sen} \theta
$$

donde $\theta$ y $\varphi$ representan la latitud y la longitud geográfica del sitio (Prescott y Hutton, 1994).

\section{Resonancia paramagnética electrónica}

Es la absorción de energía electromagnética por parte de una muestra, lo que da lugar a transiciones entre los niveles energéticos de espín electrónico generados por la aplicación de un campo magnético externo. Los electrones desapareados de los radicales libres tienen un espín $1 / 2$, y en presencia de este campo externo es posible detectar dos niveles energéticos distintos. La transición entre esos dos niveles produce una señal siempre y cuando se cumpla que

$$
h v=g \beta H
$$

donde $v$ es la frecuencia resonante, $h$ es la constante de Planck, $g$ es el llamado factor $\mathrm{g}$ o factor de Landé para electrones libres, $\beta$ es el magnetón de Bohr y $H$ es el campo magnético, lo que da cuenta del ambiente magnético local en el que está inmerso el radical. Usualmente $g$ es un tensor de nueve componentes, que pueden reducirse a dos para obtener una simetría axial, con valores asignados como $g_{\perp}$ y $g_{/ /}$, valores a los que se hace referencia cuando el campo magnético externo es perpendicular o paralelo a los ejes de simetría del sitio en el que se encuentra el centro paramagnético.

\section{Materiales y métodos}

El sitio arqueológico Checua se encuentra localizado en la vereda del mismo nombre del municipio de Nemocón (Cundinamarca), en predios de la finca Extremadura, a una distancia de 7 kilómetros al norte de la cabecera municipal. La cabecera municipal está localizada a los $5^{\circ} 04^{\prime}$ de latitud norte y $73^{\circ} 53^{\prime}$ de longitud al este de Greenwich, y a $60 \mathrm{~km}$ al noreste de Bogotá. La pieza dental utilizada en este trabajo se extrajo del entierro rotulado como Checua 2, entierro 2, encontrado a $35 \mathrm{~cm}$ de profundidad (Groot, 1992), la cual fue amablemente suministrada por el Laboratorio de Antropología Física de la Universidad Nacional de Colombia (Figura 1). El proceso de separación del esmalte se llevó a cabo siguiendo el protocolo establecido para tal fin (Kinoshita, et al., 2008; Grun, 1989; IAEA, 2002) con un equipo odontológico portátil dotado de una fresa odontológica $\mathrm{y}$ un micromotor con dispositivo de refrigeración. El calentamiento del esmalte durante el proceso de separación puede producir señales no deseables al momento de medir el espectro RPE de la muestra (IAEA, 2002), por lo que se usó agua para refrigerarla durante el proceso de separación.

El cascarón de esmalte dental se sometió a un baño ultrasónico en una solución saturada de $\mathrm{NaOH}$ al $30 \%$ durante seis horas para eliminar las impurezas orgánicas. El 
$\mathrm{NaOH}$ se retiró con agua destilada y las piezas se secaron con aire seco. Posteriormente, las muestras se sumergieron en $\mathrm{HCl}$ al $10 \%$ por 45 minutos. Además de eliminar los remanentes de dentina aún presentes, este proceso ayudó a remover las primeras capas del esmalte (del orden de $50 \pm 5$ $\mu \mathrm{m})$, lo que a su vez previno la posible contribución a la dosis total de las partículas alfa depositadas en la muestra a lo largo de su periodo de enterramiento.

Terminado el proceso, se obtuvo un cascarón de esmalte de aproximadamente $1,4 \mathrm{~mm}$, muestra que de ahora en adelante llamaremos $\mathrm{CH}-\mathrm{I}$. El esmalte se trituró en forma mecánica en un mortero ágata y el polvo resultante se dividió en nueve alícuotas, que fueron encapsuladas en tubos de plástico de aproximadamente $1,5 \mathrm{~cm}$ de largo y $0,4 \mathrm{~cm}$ de diámetro. Posteriormente se las irradió usando un equipo estándar de ${ }^{60} \mathrm{C}$, a temperatura ambiente y a $8 \mathrm{~Gy} / \mathrm{min}$ de velocidad media de dosis. Después de la irradiación, las muestras se midieron empleando un espectrómetro Bruker ESP300, banda X. Las alturas máximas de las señales $g_{\perp}$ y $g_{/ /}$se utilizaron como medida de la intensidad toda vez que su ancho entre picos permanece constante. Se obtuvieron, así, nueve valores medidos por cada dosis de radiación. Se aplicó entonces el método de dosis aditiva y el rango de irradiación fue de 0 a 740 Gy. La amplitud de la señal se graficó como función de la dosis. Los parámetros del espectrómetro fueron los siguientes: $336 \mathrm{mT}$ de campo central, 0,02 $\mathrm{T}$ de rango de barrido, 0,1 $\mathrm{mT}$ de amplitud de modulación, $100 \mathrm{kHz}$ de frecuencia de modulación, $0,328 \mathrm{~s}$ de constante de tiempo y $20 \mathrm{~mW}$ de potencia de microondas.
Dada la profundidad a la que fueron encontradas $(35 \mathrm{~cm})$, las muestras de sedimento recogidas en el lugar del hallazgo de los entierros se sometieron a un análisis radiométrico con espectrometría gama, con el fin de determinar las concentraciones en ppm de los radioisótopos ${ }^{238} \mathrm{U},{ }^{232} \mathrm{Th}$ y ${ }^{40} \mathrm{~K}$ presentes en el sitio. Para este análisis se utilizó un espectrómetro gama CANBERRA modelo GC2019 de bajo fondo, con blindaje de $15 \mathrm{~cm}$ de plomo, detector de germanio hiperpuro de alta resolución y una eficiencia absoluta de detección de 1,89 \% a 661,6 keV, perteneciente al Laboratorio de Radiometría Ambiental del Grupo de Tecnologías Nucleares de INGEOMINAS.

\section{Resultados}

La figura 2 muestra los espectros RPE de la muestra de esmalte CH-I en distintas dosis de irradiación (54 a 324 Gy). Por medio de un modelo exponencial se ajustó la curva de intensidad de la señal RPE con respecto a la dosis de irradiación (Figura 3). En la literatura normalmente se registra que las tendencias son de tipo lineal, sin embargo, hay que tener en cuenta que estos trabajos muestran resultados en un rango de dosis comprendido entre los 5 y los 100 Gy (Kinoshita, et al., 2008). El comportamiento exponencial para la señal del radical $\mathrm{CO}_{2}^{-}$, que es el que da origen a la señal RPE, se ha observado en dosis altas, de hasta $8 \mathrm{kGy}$, dependiendo de la edad de las muestras (Hoffmann, et al., 2003)). Tanto el comportamiento exponencial como el lineal son correctos y aceptables (Grun, 1989; Ikeya, 1993), sobre todo si se trabaja en un rango de dosis alto como el empleado aquí

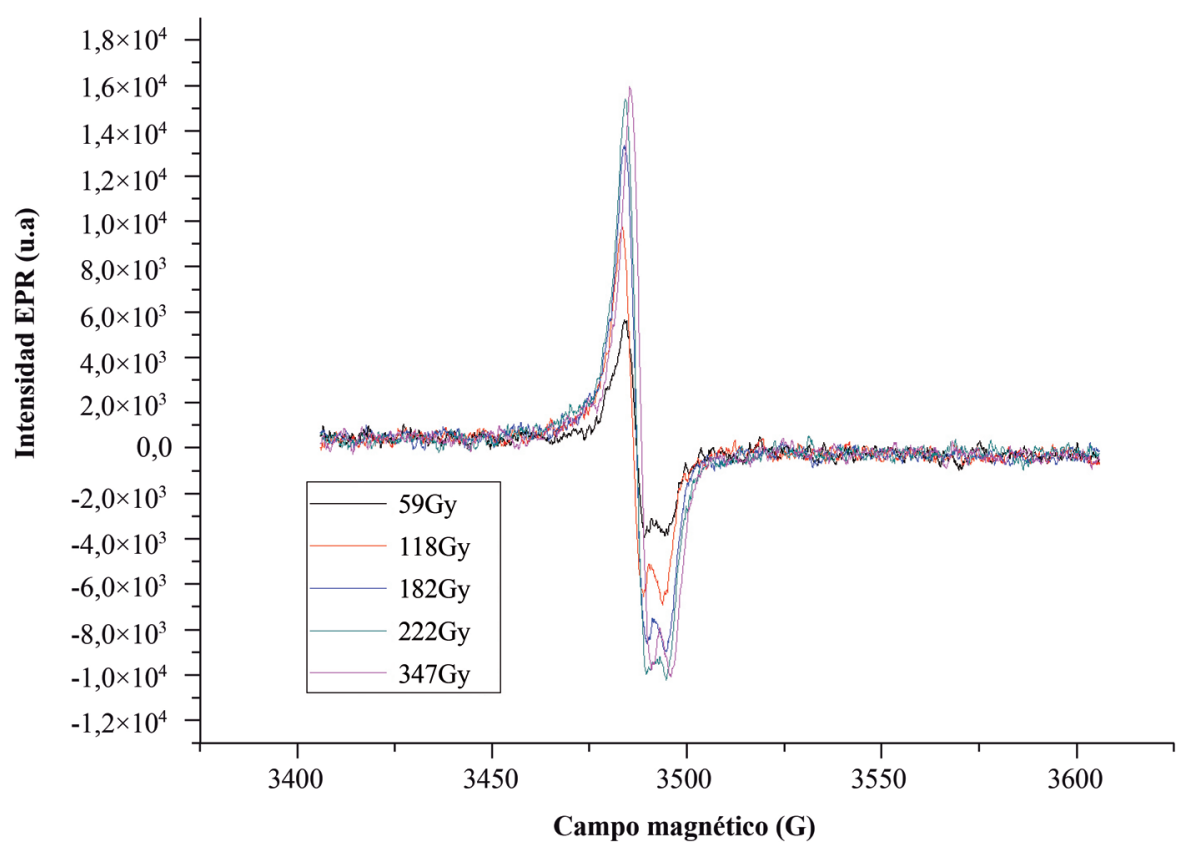

Figura 2. Espectro RPE de las muestras de esmalte dental CH-I en un rango de dosis de 59 a 347 Gy 


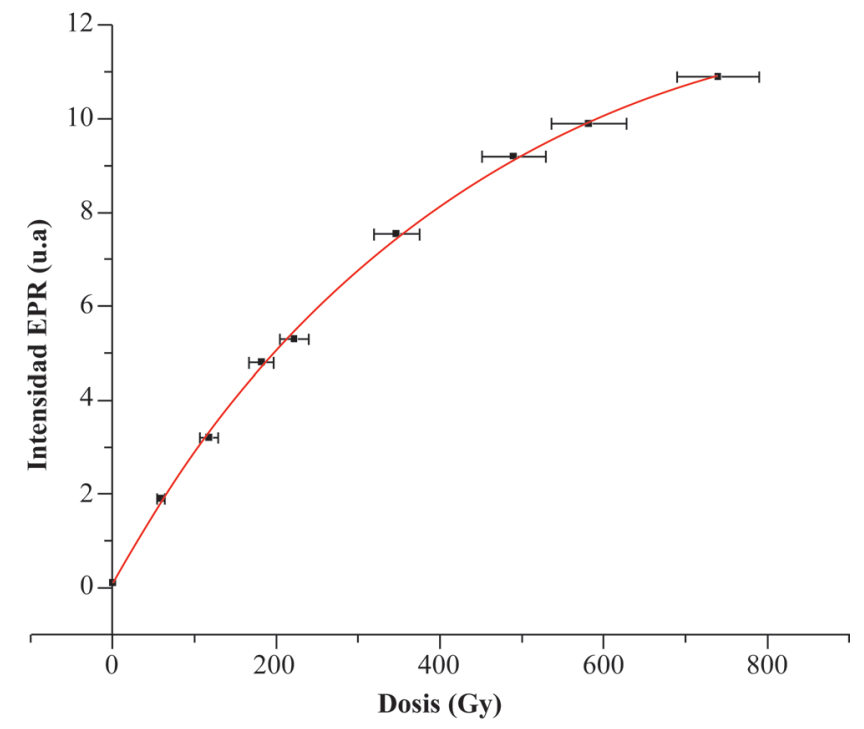

Figura 3. Intensidad de la señal RPE $V S$. dosis, que muestra el ajuste de la señal. Para la muestra CH-I, la dosis arqueológica encontrada fue la siguiente: $\mathrm{D}_{\mathrm{E}}=3,20 \pm 0,08$ Gy (coeficiente de correlación, 0,998)

para evitar mediciones hechas en los límites de saturación de las muestras. Todo lo anterior permitió obtener una dosis arqueológica o $D_{\mathrm{E}}$ de 3,20 $\pm 0,08$ Gy.

El contenido de los radioisótopos ${ }^{238} \mathrm{U},{ }^{232} \mathrm{Th} \mathrm{y}{ }^{40} \mathrm{~K}$, obtenidos a partir del análisis de suelo por espectrometría gama, se muestra en la tabla 1. En este trabajo no se determinó la concentración de estos elementos en el esmalte y la dentina, ya que su proporción se considera despreciable en muestras que datan del holoceno (Kinoshita, et al., 2008; Hefne, et al., 2002). El valor "insignificante" de estas cantidades se debe a su corto período de enterramiento, el cual se considera así en el sentido de la formación de radioisótopos dentro del esmalte y la dentina, los cuales se hallan en valores significativos más allá de los 20.000 años antes del presente (Grün, 1989; Grün, 2006; Jonas, 1997; Rink, et al., 1996), pero no así para los últimos diez mil años. La información previa suministrada por Groot (1992), en el sentido de que los habitantes del lugar del hallazgo arqueológico habrían vivido allí en la época prehispánica, entre los 8.500 y 3.000 años antes del presente, época de cazadores y recolectores en Colombia, contribuyó a la decisión de despreciar dichas cantidades.

La tasa de dosis de radiación cósmica encontrada a partir de los gráficos de Prescott y Hutton y de las ecuaciones 2, 3 y 4 (Prescott y Hutton, 1994) fue de $260 \pm 15 \mu \mathrm{Gy} / \mathrm{y}$. Otros valores, como el espesor del esmalte y la dentina (1,4 y 5 $\mathrm{mm}$, respectivamente) y las densidades del suelo, del esmalte y de la dentina $\left(2,00,3,00\right.$ y $2,82 \mathrm{~g} / \mathrm{cm}^{3}$, respectivamente), se usaron en el cálculo de la edad de la muestra. Para estos
Tabla 1. Concentraciones de uranio, de torio y de potasio determinadas por espectrometría gama en las muestras de suelo recogidas en el lugar del entierro

\begin{tabular}{cccc}
\hline \multirow{2}{*}{ Muestra } & \multicolumn{3}{c}{ Concentración (ppm) } \\
\cline { 2 - 4 } & ${ }^{238} \mathbf{U}$ & ${ }^{232} \mathbf{T h}$ & ${ }^{40} \mathbf{K}$ \\
\hline CH-I (Checua) & $1,44 \pm 0,10$ & $3,78 \pm 0,23$ & $0,77 \pm 0,05$ \\
\hline
\end{tabular}

Tabla 2. Tasa de dosis obtenidas a partir de la concentración de radioisótopos en el suelo, radiación cósmica local y tasa de dosis total (valores reportados por el programa ROSY)

\begin{tabular}{lccccc}
\hline & $\begin{array}{c}\text { Alpha } \\
(\boldsymbol{\mu G y} / \mathbf{y})\end{array}$ & $\begin{array}{c}\text { Beta } \\
(\mu \mathrm{Gy} / \mathbf{y})\end{array}$ & $\begin{array}{c}\text { Gama } \\
(\boldsymbol{\mu G y} / \mathbf{y})\end{array}$ & $\begin{array}{c}\text { Cósmica } \\
(\boldsymbol{\mu} \mathbf{G y} / \mathbf{y})\end{array}$ & $\begin{array}{c}\text { Gama + cósmica } \\
(\boldsymbol{\mu} \mathbf{G y} / \mathbf{y})\end{array}$ \\
\hline Dentina & 0,00 & $0,00-$ & - & - & - \\
Esmalte & 0,00 & 0,00 & - & - & - \\
Cemento & 0,00 & 0,00 & - & - & - \\
Sedimento & 0,00 & 43,34 & 342,65 & - & - \\
Uranio & 0,00 & 29,76 & 162,72 & - & - \\
Torio & 0,00 & 13,58 & 179,93 & - & - \\
Potasio & - & 0,00 & 0,00 & - & - \\
Total & 0,00 & 43,34 & 342,65 & 260,00 & 598,31 \\
Tasa de dosis Alpha+beta $(\mu \mathrm{Gy} / \mathrm{y})=43,34$ \\
\multicolumn{7}{l}{ Tasa de dosis total $(\mu \mathrm{Gy} / \mathrm{y})=645,99$} \\
\hline
\end{tabular}

cálculos también se hace necesario conocer el contenido de agua en el suelo del lugar del entierro, que en nuestro caso fue de $12,1 \%$.

El programa computacional ROSY se usó para calcular las tasas de dosis de radiación debidas a cada uno de los radioisótopos y sus respectivas concentraciones. La tasa de dosis de radiación total, $\angle D>$, debida al medio y a la radiación cósmica, e independiente del modelo de absorción de uranio, fue de $645,99 \mu \mathrm{Gy} / \mathrm{y}$. Todos los resultados se muestran en la tabla 2. Se emplearon algunos otros datos necesarios para determinar estas tasas de dosis, valores que el programa ROSY aporta por defecto. Así pues, considerando la ecuación 1, la edad media de la muestra CH-I fue de $5.021 \pm 202$ años antes del presente.

\section{Conclusiones}

La edad reportada para muchas muestras arqueológicas encontradas en Colombia se ha determinado a partir del ${ }^{14} \mathrm{C}$. Este no es un método de datación directo, toda vez que lo que se data es el material orgánico encontrado alrededor del entierro, el cual puede coincidir cronológicamente o no con los restos humanos descubiertos. De allí la importancia de los resultados publicados en este trabajo con relación a la datación directa de un individuo arqueológico de nuestro territorio mediante la resonancia paramagnética electrónica (RPE). La edad media encontrada fue de 5.021 \pm 202 años antes del presente, resultado que concuerda con los análisis estratigráficos del sitio publicados por Groot (1992). 
La edad calculada para el individuo bajo estudio, perteneciente a un entierro hallado en territorio colombiano, se hizo en forma relativamente sencilla, con unos pocos gramos de esmalte dental obtenido de un único molar, lo que evidencia las ventajas y características de la datación por RPE. Esperamos que los resultados aquí presentados contribuyan a unir otros eslabones de la larga cadena de la historia colombiana y americana, en la cual aún hay mucho por hallar debido al rico pasado prehispánico y colonial de nuestros países. Este trabajo representa, pues, un esfuerzo pionero destinado a promover la datación de nuestros antepasados.

\section{Agradecimientos}

Los autores agradecemos a la Dirección de Investigación, sede Bogotá, de la Universidad Nacional de Colombia, por el apoyo financiero a través del proyecto 8003348 . Agradecemos a M. Peña, de INGEOMINAS, por el análisis de suelos, así como al Laboratorio de Antropología de la Universidad Nacional de Colombia, en especial a su director, el doctor J.V. Rodríguez, por suministrarnos la pieza dental estudiada, y a la doctora Ana M. Groot, por sus comentarios durante la investigación.

\section{Conflicto de interés}

Los autores declaran que no tienen ningún conflicto de interés.

\section{Bibliografía}

Carvajal E, Montes L., Almanza O. A. (2011). Quaternary dating by electron spin resonance (ESR) applied to human tooth enamel. Earth Sci. Res. SJ. 15 (2): 115 - 120.

Carvajal, E. (2011). Datación de esmalte dental de restos fósiles humanos provenientes de Aguazuque y Checua (Cundinamarca) usando la técnica de resonancia paramagnética electrónica (EPR). Tesis de Maestría en Física, Universidad Nacional, Bogotá, Colombia. pp. 73-78.

Groot, A.M. (1992). Checua. Una secuencia cultural entre 8500 y 3000 años antes del presente. Fundación de Investigaciones Arqueológicas Nacionales. Banco de la República. Bogotá. pp. $17-80$.

Grün, R. (1989). Electron Spin Resonance (ESR) dating. Quaternary International 1: 65-109.
Grün, R. (2006). Direct Dating of Human Fossils. Yearb. Phys. Anthropol. 49: 2-48.

Hefne, J., Yamani, A., Al-Dayel, O., Ikeya, M., Al-Osaimi, S. (2002). ESR Dating of Tooth from Pre-Islamic Sitin Saudi Arabia. Advances in ESR Applications. 18: 119-121.

Hoffmann D, Woda C, Mangini A. (2003). Equivalent Dose Determination in Foraminifera: Analytical description of the CO2-signal Dose-Response Curve. Radiation Measurements 37: 95-101.

IAEA (2002). Use of electron paramagnetic resonance dosimetry with tooth enamel for retrospective dose assessment. Report of a coordinated research project. IAEA-TECDOC-1331. Vienna.

Ikeya, M. (1993). New applications of electron spin resonance: Dating, dosimetry and microscopy, World Scientific Publishing Company, Singapore. 2: 66-102.

Jonas, M. (1997). Concepts and Methods of ESR dating. Radiat Meas. 27: 943-973.

Kinoshita, A., Figueiredo, A.M.G., Felice, G.D., Lage, M.C.S.M., Guidon, N., Baffa, O. (2008). Electron spin resonance dating of human teeth from Toca da Santa shelter of São Raimundo Nonato, Piauí, Brazil. Nucl. Instrum. Meth B. 266: 635-639.

Prescott, J.R., Hutton, J.T. (1994). Cosmic ray contributions to dose-rates for luminescense and ESR dating: Large depths and long-term time variations. Radiat. Meas. 23: 497-500.

Renfrew, C. y Bahn, P. Arqueología. Teorías, Métodos y Práctica (1992): Ediciones Akal S.A. pp. 107-147.

Sandoval, J. (2010). Datación de restos humanos prehispánicos a través de esmalte dental usando resonancia paramagnética electrónica (EPR). Trabajo de grado en Antropología, Universidad Nacional, Bogotá, Colombia. pp. 60-77

Watanabe S., Feria Ayta, W. E., Hamaguchi, H. (2003). Some Evidence of a Date of First Humans to Arrive in Brazil. Journal of Archaeological Science. 30: 351-354.

Ülkü U. (2004). ESR studies of Anatolian gypsum. Spectrochimica Acta Part A. 60: 1359-1365.

Walker M. (2005). Quaternary Dating Methods. John Wiley \& Sons Ltd. The Atrium, Southern Gate, Chichester. Chapters 1, 2, 3 and 4. pp. 17-34 